\title{
A simple method to compute volumes of even-dimensional Coxeter polyhedra
}

\author{
Julien Paupert *
}

May 7, 2012

\section{Introduction}

Understanding and computing volumes of hyperbolic manifolds and orbifolds is a rich and fascinating subject. There are for instance deep connections with number theory, more specifically special values of arithmetic functions such as Dedekind $\zeta$-functions, Dirichlet $L$-functions and polylogarithms (see [Za], [K2], Prasad's volume formula from $[\mathrm{P}]$ as used in the hyperbolic case in $[\mathrm{Be}]$ and $[\mathrm{BE}])$.

In general, finding the volume of a hyperbolic manifold or orbifold is a difficult problem. However in even dimensions the Gauss-Bonnet-Chern theorem asserts that hyperbolic volume is a multiple of Euler-Poincaré characteristic (see section 3). Therefore, if one can compute the Euler characteristic of a hyperbolic manifold or orbifold (for instance if one knows a cell decomposition for it) then its volume is easily computed. Hyperbolic Coxeter groups form a large class of groups for which a cell decomposition of the quotient orbifold is known, and is in fact contained in the Coxeter diagram data (see sections 2 and 3). Indeed, by results of Vinberg, the faces of a hyperbolic Coxeter polyhedron correspond to the elliptic subdiagrams of its Coxeter diagram, with such a subdiagram giving the stabilizer of the corresponding face. Moreover, hyperbolic Coxeter polyhedra are simple (in the sense that links of faces are simplices), so that the dimension of each face is as expected (in other words, the walls of the polyhedron intersecting along that face are in general position). This means that one can compute the (orbifold) Euler characteristic of the quotient of hyperbolic space $\mathrm{H}^{n}$ by a hyperbolic Coxeter group $\Gamma$ by the formula:

$$
\chi^{\text {orb }}\left(\Gamma \backslash \mathrm{H}^{n}\right)=\sum_{D_{\Gamma^{\prime}}} \frac{(-1)^{n-\left|D_{\Gamma^{\prime}}\right|}}{\left|\Gamma^{\prime}\right|}
$$

where $D_{\Gamma}$ is the Coxeter diagram of $\Gamma$, and the sum is taken over all elliptic subdiagrams $D_{\Gamma^{\prime}}$ (not necessarily connected) of $D_{\Gamma}$. One then obtains in even dimensions $n=2 \mathrm{~m}$ the volume of the corresponding Coxeter polyhedron $P_{\Gamma}$ by the Gauss-Bonnet-Chern theorem:

$$
\operatorname{Vol}\left(P_{\Gamma}\right)=C(n) \chi\left(\Gamma \backslash \mathrm{H}^{n}\right)=(-1)^{m} \frac{\pi^{m} 2^{n} m !}{n !} \chi^{\text {orb }}\left(\Gamma \backslash \mathrm{H}^{n}\right)
$$

\footnotetext{
*Author supported by NSF grant DMS 1007340.
} 
We give the necessary background on Coxeter groups, their polyhedra and diagrams in section 2, on Euler characteristic and volume in section 3, and give examples of computations in low dimensions (4 and 6) in section 4.

These results are all well-known, especially to specialists; see for instance [ACT], and [K1], [Ze] for a related method, more complicated but more efficient. The latter uses Schläfli's reduction formula, which only requires knowing the faces whose dimension is of same parity as the dimension of the polyhedron (as opposed to the Euler characterstic, which requires knowing all faces). This becomes more and more relevant as the dimension increases, and in practice we find it better in dimensions 8 and above.

The point of this note is to give a self-contained exposition of this simple case, accessible to a wider mathematical audience. Moreover the volume computations for the non-arithmetic examples $\Gamma_{4}$ and $\Gamma_{5}$ in dimensions 4 and 6 found in sections 4.2 and 4.3 are new, as far as I know. The volume of the polyhedron corresponding to $\Gamma_{3}$ was computed in [K1].

I would like to thank Daniel Allcock for providing a copy of Ruzmanov's paper [R], with translation by Eugene Tevelev.

\section{Coxeter polyhedra and diagrams}

Let $X$ denote one of the 3 model spaces of constant curvature, $X=\mathrm{S}^{n}, \mathrm{E}^{n}$ or $\mathrm{H}^{n}$. A polyhedron in $X$ is a subset $P$ of $X$ which is bounded by finitely many hyperplanes, in other words $P$ is the intersection of finitely many closed half-spaces. A wall of $P$ is a hyperplane $H$ such that $P \cap H$ has dimension $n-1$. In that case we will say that $P \cap H$ is a (maximal) face of $P$; in turn this polyhedron of dimension $n-1$ has faces, and inductively this defines the set of all faces of $P$. (Alternatively, in such a constant curvature space one can also construct $P$ from the bottom up, by taking convex hulls of vertices). To avoid all confusion we will call $k$-face of $P$ any of its faces of dimension $k$.

A Coxeter polyhedron in $X$ is a polyhedron $P$ all of whose dihedral angles are submultiples of $\pi$. (This means that if $H_{i}$ and $H_{j}$ are 2 walls of $P$, then either they are disjoint or at any intersection point their outward-pointing normals form an angle of measure $\pi-\pi / m_{i j}$ where $m_{i j} \in \mathbb{N} \cup\{\infty\}$ ). The point of this condition is that the reflections in the walls of such a polyhedron generate a discrete group of isometries of $X$, for which $P$ is a fundamental polyhedron. More explicitly, if $S_{1}, \ldots, S_{k}$ denote the walls of $P$ and $r_{1}, \ldots, r_{k}$ the reflections in them, then $\Gamma=\left\langle r_{1}, \ldots, r_{k}\right\rangle$ is a discrete subgroup of $\operatorname{Isom}(X)$. In fact such a $\Gamma$ is a Coxeter group in the sense that it admits the presentation (adopting the convention that $m_{i i}=2$ for all $i)$ :

$$
\left.\Gamma=\left\langle r_{1}, \ldots, r_{k}\right|\left(r_{i} r_{j}\right)^{m_{i j}}=1(\text { for } 1 \leqslant i \leqslant j \leqslant k)\right\rangle
$$

A convenient way to encode the information about such a Coxeter group $\Gamma$ is by means of the associated Coxeter diagram $D_{\Gamma}([\mathrm{Co}])$ which is a graph with labeled edges, constructed as follows. The vertices of $D_{\Gamma}$ correspond to the generating reflections $r_{1}, \ldots, r_{k}$; two distinct vertices corresponding to $r_{i}$ and $r_{j}$ are joined by an edge if (a) $m_{i j} \geqslant 3$, in which case the edge is labeled $m_{i j}$ (alternatively, an edge of multiplicity $m_{i j}-2$ is drawn), or (b) by a bold (resp. dotted) edge if the walls of $r_{i}$ and $r_{j}$ are parallel (resp. ultraparallel), in the hyperbolic case. The point of this convention is that connected components of $D_{\Gamma}$ correspond to irreducible factors of $\Gamma$. Examples of Coxeter diagrams can be found in section 4 . 
The numbers $m_{i j}$ also determine a quadratic form via a symmetric matrix called the Gram matrix of the polyhedron or group. Namely, if $\Gamma$ is generated by the $k$ reflections $r_{1}, \ldots, r_{k}$ with $m_{i j}$ as above, then the Gram matrix of $\Gamma$ is the symmetric $k \times k$ matrix with 1 's on the diagonal and $(i, j)$-entry equal to $-\cos \left(\pi / m_{i j}\right)$ (resp. $-\cosh d\left(H_{i}, H_{j}\right)$ if the walls $H_{i}$ and $H_{j}$ are parallel or ultraparallel). This is the Gram matrix of the outward-pointing normal vectors to the walls of $P$. One can then define invariants such as the determinant, rank, signature of $P$ ( or $\Gamma$ or $D_{\Gamma}$ ) as the corresponding invariant of its Gram matrix. Accordingly, we will say that $D_{\Gamma}$ is hyperbolic if its Gram matrix is of signature $(n, 1)$ for some $n \geqslant 1$, parabolic if it is positive semidefinite (but not definite), and elliptic if it is positive definite. Note that elliptic and parabolic Coxeter diagrams were classified by Coxeter in 1934 ([Co]), whereas no such classification is known for hyperbolic diagrams (except the case of hyperbolic simplex groups and various cases with fixed number of maximal faces, as in $[\mathrm{T}]$ ).

Vinberg has however proved the following general existence result for acute-angled hyperbolic polyhedra (Theorem 2.1 of [V2]):

Theorem 2.1 (Vinberg) Let $G$ be an indecomposable symmetric matrix of signature $(n, 1)$ with 1's on the diagonal and non-positive entries off it. Then there exists a convex polyhedron in $\mathrm{H}^{n}$ whose Gram matrix is $G$, and this polyhedron is unique up to isometry.

The Gram matrix represents a quadratic form on $\mathbb{R}^{k}$ which is preserved by the so-called "geometric representation" (by Bourbaki, [Bo] and Humphreys, [Hu]). More relevant from our point of view is the representation that we started with (of $\Gamma$ in $\operatorname{Isom}\left(\mathrm{H}^{n}\right)<\operatorname{PGL}(n+1, \mathbb{R})$ arising from the polyhedron $P$ in $\mathrm{H}^{n}$ ). These representations coincide only when the polyhedron is a simplex, that is when the Gram matrix has maximal rank.

\section{Euler characteristic and volume}

Let $M$ be a manifold modelled on $X$, in the sense that $M=\Gamma \backslash X$, with $\Gamma$ a (torsion-free) discrete subgroup of $\operatorname{Isom}(X)$. Then $M$ inherits a volume form $d \mathrm{Vol}$ from that of $X$. In the case where $n=\operatorname{dim} X$ is even $(n=2 m)$ and $M$ is compact, the celebrated Gauss-BonnetChern theorem (see $[\mathrm{Sp}])$ asserts that:

$$
\int_{M} K_{n} d \mathrm{Vol}=\frac{\pi^{m} 2^{n} m !}{n !} \chi(M)
$$

where $K_{n}$ denotes the Gaussian curvature of $M$ (the product of principal curvatures) and $\chi(M)$ the Euler characteristic of $M$. In the constant curvature case one has $K_{n}=1$ for $X=\mathrm{S}^{n}, K_{n}=0$ when $X=\mathrm{E}^{n}$ and $K_{n}=(-1)^{m}$ when $X=\mathrm{H}^{2 m}$. Focusing on the hyperbolic case, one obtains for a compact hyperbolic manifold of even dimension $n=2 m$ :

$$
\operatorname{Vol}(M)=C(n) \chi(M)=(-1)^{m} \frac{\pi^{m} 2^{n} m !}{n !} \chi(M)
$$

This proportionality relation between volume and Euler characteristic has been extended in several directions:

- for noncompact, finite-volume hyperbolic manifolds, (1) holds as is (see [G] and [KZ]). 
- in the complex hyperbolic case (where $X=\mathrm{H}_{\mathbb{C}}^{n}$, the simply connected manifold with a Kähler metric of constant holomorphic curvature, but nonconstant sectional curvature), relation 1 holds with a different constant, $C(n)=(-1)^{m} \frac{\pi^{m} 2^{n}}{(m+1) !}$. In this setting it is also known as the Hirzebruch proportionality principle (see [Hi] and $[\mathrm{M}]$ ).

- when $M$ is only an orbifold, i. e. a finite quotient of a manifold (alternatively, in our setting, $M=\Gamma \backslash X$, with $\Gamma$ a discrete subgroup of $\operatorname{Isom}(X)$ which may have torsion), relation (1) also holds, replacing $\chi(M)$ with $\chi^{\text {orb }}(M)$, the so-called orbifold Euler characteristic, which is simply defined by extending $\chi$ to orbifolds by declaring that it is multiplicative on covers. (The orbifold version of (1) then follows, as volume is also multiplicative on covers).

In practice the orbifold Euler characteristic can be computed from a cellular decomposition of $M$ in much the same way as the usual Euler characteristic. However one needs more information, namely the orders of the face stabilizers in the orbifold fundamental group $\Gamma$. More precisely, let $M=\Gamma \backslash X$, with $\Gamma$ a discrete subgroup of $\operatorname{Isom}(X)$, and suppose that $P \subset X$ is a fundamental polyhedron for the action of $\Gamma$ on $X$. In particular, the maximal faces of $P$ are identified in pairs by elements of $\Gamma$ (the side-pairings), and these side-pairings induce identifications of the lower-dimensional faces of $P$ as well. Then $M$ is homeomorphic (and in fact isometric) to the quotient space of $P$ by the face identifications.

For $k=0, \ldots, n$ denote by $\mathcal{F}_{k}$ the set of orbits of $k$-faces of $P$ under $\Gamma$ (these are the faces of the cellular decomposition of $M$ ). Two faces in the same orbit have conjugate stabilizers in $\Gamma$; in particular the order of this stabilizer is well-defined along orbits. It can then easily be checked that by the above definition:

$$
\chi^{o r b}(M)=\sum_{k=0}^{n} \sum_{F \in \mathcal{F}_{k}} \frac{(-1)^{k}}{\left|\operatorname{Stab}_{\Gamma}(F)\right|}
$$

For Coxeter polyhedra the maximal faces are paired with themselves and there are no nontrivial face identifications. This means that in practice, one only needs to know the orders of the face stabilizers. This can be directly read from the Coxeter diagram by the following fact, due to Vinberg (Theorems 3.1 of [V2]):

Theorem 3.1 (Vinberg) The faces of a hyperbolic Coxeter polyhedron are in one-to-one correspondence with the elliptic subdiagrams of its Coxeter diagram. Moreover the elliptic subdiagram corresponding to a given face is the Coxeter diagram of its stablilizer, and the order of the subdiagram is the codimension of the face.

This result implies the following expression for the orbifold Euler characteristic of a hyperbolic Coxeter group $\Gamma$ of rank $n+1$, where the sum is taken over all elliptic subdiagrams $D_{\Gamma^{\prime}}$ (not necessarily connected) of $D_{\Gamma}$ :

$$
\chi^{\text {orb }}\left(\Gamma \backslash \mathrm{H}^{n}\right)=\sum_{D_{\Gamma^{\prime}}} \frac{(-1)^{n-\left|D_{\Gamma^{\prime}}\right|}}{\left|\Gamma^{\prime}\right|}
$$


Figure 1: Coxeter diagram for $\Gamma_{1}$

\section{Examples}

In practice, given a Coxeter diagram $D$ we need a systematic way of listing all elliptic subdiagrams of $D$ (the dangers are forgetting any or overcounting). We have chosen to remove vertices from right to left, in lexicographic order (adapting the ordering of vertices slightly when $D$ has branch points). We give all the details in the first example (in the form of a table), the others being analogous. We start with the simplest case of a simplex in $\mathrm{H}^{4}$, with one compact and one non-compact example, then give two 4-dimensional and one 6-dimensional non-arithmetic examples.

\subsection{Simplices in $\mathrm{H}^{4}$}

We consider the group $\Gamma_{1}$ whose Coxeter diagram is pictured in Figure 2; the corresponding polyhedron is a compact simplex in $\mathrm{H}^{4}$. Its volume was first computed in [K1].

\begin{tabular}{|l|l|l|}
\hline Dimension of face & Elliptic subdiagram & Order of face stabilizer \\
\hline 0 & $H_{4}$ & 14400 \\
& $H_{3} \times \bullet$ & 240 \\
& $G_{2}(5) \times A_{2}$ & 60 \\
& $\bullet \times A_{3}$ & 48 \\
& $A_{4}$ & 120 \\
\hline 1 & $H_{3}$ & 120 \\
& $G_{2}(5) \times \bullet$ & 20 \\
& $G_{2}(5) \times \bullet$ & 20 \\
& $\bullet \times A_{2}$ & 12 \\
& $\bullet \times \bullet \times \bullet$ & 8 \\
& $\bullet \times A_{2}$ & 12 \\
& $A_{3}$ & 24 \\
& $A_{2} \times \bullet$ & 12 \\
& $\bullet \times A_{2}$ & 12 \\
& $A_{3}$ & 24 \\
\hline 2 & $G_{2}(5)$ & 10 \\
& $\bullet \times \bullet$ & 4 \\
& $A_{2}$ & 6 \\
& $\bullet \times \bullet$ & 4 \\
& $\bullet \times \bullet$ & 4 \\
& $A_{2}$ & 6 \\
& $\bullet \times \bullet$ & 4 \\
& $\bullet \times \bullet$ & 4 \\
& $\bullet \times \bullet$ & 4 \\
& $A_{2}$ & 6 \\
\hline 4 & $\bullet(5$ times $)$ & 2 \\
\hline
\end{tabular}


Figure 2: Coxeter diagram for $\Gamma_{2}$

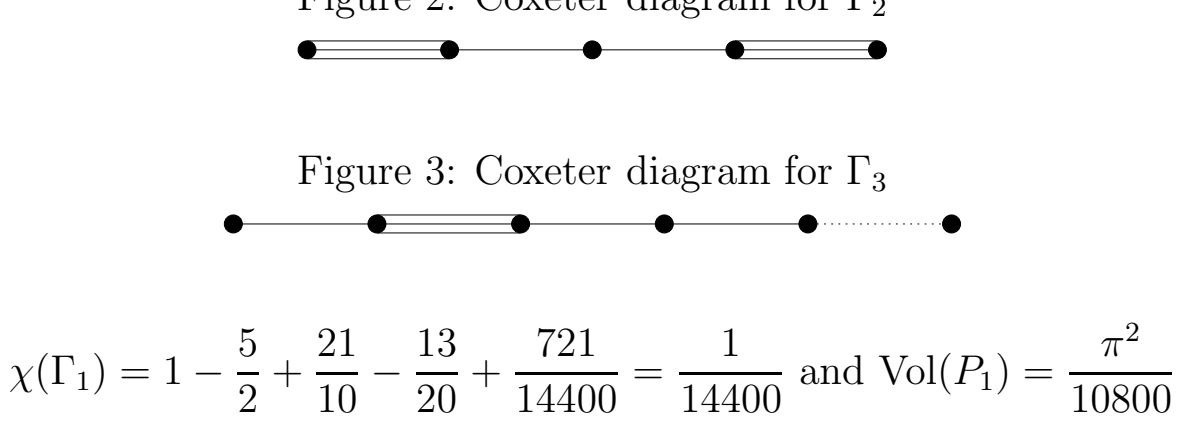

We now consider the group $\Gamma_{2}$ whose Coxeter diagram is pictured in Figure 2; the corresponding polyhedron is a non-compact simplex in $\mathrm{H}^{4}$. Its volume was first computed in $[\mathrm{K} 1]$.

$$
\chi\left(\Gamma_{2}\right)=1-\frac{5}{2}+\frac{61}{30}-\frac{11}{20}+\frac{133}{7200}=\frac{13}{7200} \text { and } \operatorname{Vol}\left(P_{2}\right)=\frac{13 \pi^{2}}{5400}
$$

\subsection{Non-arithmetic examples in $\mathrm{H}^{4}$}

The groups $\Gamma_{3}$ and $\Gamma_{4}$ are examples of non-arithmetic Coxeter groups in $\operatorname{Isom}\left(\mathrm{H}^{4}\right)$ taken from [V1], the former cocompact and the latter non-cocompact (see Coxeter diagrams in Figures 3 and 4 ). The covolume of $\Gamma_{3}$ was first computed in [K1].

$$
\begin{aligned}
& \chi\left(\Gamma_{3}\right)=1-\frac{6}{2}+\frac{31}{10}-\frac{149}{120}+\frac{2081}{14400}=\frac{41}{14400} \text { and } \operatorname{Vol}\left(P_{3}\right)=\frac{41 \pi^{2}}{10800} \\
& \chi\left(\Gamma_{4}\right)=1-\frac{6}{2}+\frac{367}{120}-\frac{278}{240}+\frac{1531}{14400}=\frac{91}{14400} \text { and } \operatorname{Vol}\left(P_{4}\right)=\frac{91 \pi^{2}}{10800}
\end{aligned}
$$

\subsection{A non-arithmetic example in $\mathrm{H}^{6}$}

In $[R]$ Ruzmanov constructed examples of non-arithmetic finite-volume Coxeter polyhedra in $\mathrm{H}^{n}$ for $6 \leqslant n \leqslant 10$. We now compute the Euler characteristic of his 6 -dimensional Coxeter group, which we denote $\Gamma_{5}$ (see Coxeter diagram in Figure 5) This group also appears in [T] as it has $8=6+2$ maximal faces.

Figure 4: Coxeter diagram for $\Gamma_{4}$ 
Figure 5: Coxeter diagram for $\Gamma_{5}$

\begin{tabular}{|c|c|c|}
\hline Dimension of face & Elliptic subdiagram & Order of face stabilizer \\
\hline \multirow[t]{9}{*}{0} & $A_{5} \times \bullet$ & 1440 \\
\hline & $A_{4} \times A_{2}$ & 720 \\
\hline & $A_{3} \times B_{3}$ & 1152 \\
\hline & $A_{2} \times B_{4}$ & 2304 \\
\hline & $A_{2} \times F_{4}$ & 6912 \\
\hline & & 46080 \\
\hline & $B_{5} \times \bullet$ & 7680 \\
\hline & $B_{3} \times B_{3}$ & 2304 \\
\hline & $B_{2} \times F_{4}$ & 9216 \\
\hline \multirow[t]{13}{*}{1} & $A_{5}$ & 720 \\
\hline & $A_{4} \times \bullet(3$ times $)$ & 240 \\
\hline & $A_{3} \times A_{2}($ twice $)$ & 144 \\
\hline & $A_{3} \times \bullet^{2}(3$ times $)$ & 96 \\
\hline & $A_{3} \times B_{2}$ & 192 \\
\hline & $A_{2} \times A_{2} \times \bullet(4$ times $)$ & 72 \\
\hline & $A_{2} \times B_{3}(4$ times $)$ & 288 \\
\hline & $A_{2} \times B_{2} \times \bullet($ twice $)$ & 96 \\
\hline & $B_{5}$ (twice) & 3840 \\
\hline & $B_{4} \times \bullet(4$ times $)$ & 768 \\
\hline & $B_{3} \times B_{2}$ (twice) & 384 \\
\hline & $B_{3} \times \bullet^{2}(4$ times $)$ & 192 \\
\hline & $F_{4} \times \bullet(3$ times $)$ & 2304 \\
\hline \multirow[t]{11}{*}{2} & $A_{4}($ twice $)$ & 120 \\
\hline & $A_{3} \times \bullet(8$ times $)$ & 48 \\
\hline & $A_{2} \times A_{2}(4$ times $)$ & 36 \\
\hline & $A_{2} \times B_{2}(4$ times $)$ & 48 \\
\hline & $A_{2} \times \bullet^{2}(16$ times $)$ & 24 \\
\hline & $\bullet^{4}$ (twice) & 16 \\
\hline & $B_{4}($ twice $)$ & 384 \\
\hline & $B_{3} \times \bullet(10$ times $)$ & 768 \\
\hline & $B_{2} \times B_{2}$ & 64 \\
\hline & $B_{2} \times \bullet^{2}(5$ times $)$ & 32 \\
\hline & $F_{4}$ & 1152 \\
\hline \multirow[t]{5}{*}{3} & $A_{3}(3$ times $)$ & 24 \\
\hline & $A_{2} \times \bullet(20$ times $)$ & 12 \\
\hline & $\bullet^{3}(16$ times $)$ & 8 \\
\hline & $B_{3}(3$ times $)$ & 48 \\
\hline & $B_{2} \times \bullet(8$ times $)$ & 16 \\
\hline \multirow[t]{3}{*}{4} & $A_{2}(5$ times $)$ & 6 \\
\hline & $\bullet^{2}(20$ times $)$ & 4 \\
\hline & $B_{2}$ (twice) & 8 \\
\hline 5 & - $(8$ times $)$ & 2 \\
\hline 6 & $\emptyset$ & 1 \\
\hline
\end{tabular}




$$
\chi\left(\Gamma_{5}\right)=1-\frac{8}{2}+\frac{73}{12}-\frac{209}{48}+\frac{653}{480}-\frac{2161}{11520}+\frac{311}{86400}=-\frac{16313}{172800} \text { and } \operatorname{Vol}\left(P_{5}\right)=\frac{16313 \pi^{3}}{324000}
$$

\section{References}

[ACT] D. Allcock, J. Carlson, D. Toledo; Real cubic surfaces and real hyperbolic geometry. C. R. Math. Acad. Sci. Paris 337 (2003), 185-188.

[Be] M. Belolipetsky; On the volumes of arithmetic quotients of $S O(1, n)$. Ann. Scuola Norm. Sup. Pisa Cl. Sci. (5) III (2004) 749-770.

[BE] M. Belolipetsky, V. Emery; On volumes of arithmetic quotients of $P O(1, n)^{0}, n$ odd. Preprint 2010.

[Bo] N. Bourbaki; Groupes et algèbres de Lie, Ch. 4-6. Hermann, Paris (1968).

[Ch] I.M. Chiswell; The Euler characteristic of graph products and of Coxeter groups. Discrete groups and geometry (Birmingham 1991), 36-46, London Math. Soc. Lecture Note Ser. 173, Cambridge Univ. Press, 1992.

[Co] H.S.M. Coxeter. Discrete groups generated by reflections. Ann. Math. 35 (1934), 588621.

[G] M. Gromov; Volume and bounded cohomology. Inst. Hautes Études Sci. Publ. Math. 56 (1982), 5-99.

[Hi] F. Hirzebruch; Topological methods in Algebraic Geometry (3d ed). Grundlehren Math. Wiss. 131, Springer-Verlag, New York (1966).

[Hu] J.E. Humphreys; Reflection Groups and Coxeter Groups. Cambridge studies in advanced mathematics 29. Cambridge University Press, Cambridge (1990).

[JKRT] N.W. Johnson, R. Kellerhals, J.G. Ratcliffe, S.T. Tschantz; The size of a hyperbolic Coxeter simplex. Transf. Groups 4 (1999), 329-353.

[K1] R. Kellerhals; On Schläfli's reduction formula. Math. Z. 206 (1991), 193-210.

[K2] R. Kellerhals; The dilogarithm and volumes of hyperbolic polytopes. Structural properties of polylogarithms, 301-336. Math. Surveys Monogr. 37, AMS, Providence (1991).

[KZ] R. Kellerhals, T. Zehrt; The Gauss-Bonnet formula for hyperbolic manifolds of finite volume. Geom. Dedicata 84 (2001), 49-62.

[M] D. Mumford; Hirzebruch's proportionality theorem in the noncompact case. Invent. Math. 42 (1977), 239-272.

[P] G. Prasad; Volumes of S-arithmetic quotients of semi-simple groups. Publ. Math. IHES 69 (1989), 91-117.

[R] O.P. Ruzmanov; Examples of non-arithmetic crystallographic Coxeter groups in the $n$ dimensional Lobachevskij space for $6 \leqslant n \leqslant 10$. Vopr. Teor. Grupp. Gomologicheskoy Algebry 9 (1989), 138-142 (Russian). 
[Se] J.-P. Serre; Cohomologie des groupes discrets. Prospects in mathematics, 77-169, Ann. of Math. Studies 70, Princeton Univ. Press, 1971.

[Sp] M. Spivak; A comprehensive introduction to Differential Geometry. Vol. 5. Publish or Perish.

[T] P. Tumarkin; Hyperbolic Coxeter n-polytopes with $n+2$ facets. Math. Notes 75 (6) (2004), 848-854.

[V1] E.B. Vinberg; Geometry II, in: Encyclopedia of Mathematical Sciences, vol. 29, Springer-Verlag, 1993.

[V2] E. B. Vinberg; Hyperbolic reflection groups. Russian Math. Surveys 40:1 (1985), 29-66.

[Za] D. Zagier; Hyperbolic volumes and special values of Dedekind zeta-functions. Invent. Math. 83 (1986), no. 2, 285-301.

[Ze] T. Zehrt; The covolume of discrete subgroups of $I s o\left(\mathbb{H}^{2 m}\right)$ Discrete Mathematics 309 (2009) 2284-2291. 